\title{
Numerical Simulation of HFCVD Process Used for Diamond Growth
}

\author{
Divani C. Barbosa*, Hélcio F. V. Nova ${ }^{\dagger}$, and Mauricio R. Baldan* \\ Instituto Nacional de Pesquisas Espaciais, \\ *Laboratório Associado de Sensores e Materiais and \\ ${ }^{\dagger}$ Laboratório Associado de Computação e Matemática Aplicada, \\ Caixa Postal 515, 12245-970 São José dos Campos-SP, Brazil
}

Received on 4 April, 2005

\begin{abstract}
Hot-filament chemical vapour deposition (HFCVD) is a common method employed for diamond deposition. Typically in this method a dilute mixture of carbon containing gas such as methane in hydrogen is thermally activated at sub atmospheric pressures by a hot filament. Due to the filament-substrate proximity, large temperature variation across the substrate is possible. In this work we investigate the role of fluid flow and heat transfer from the filament to substrate in determining the quality of diamond growth. The commercial software CFX was used to calculate velocity field, temperature distribution and fluid flow. A vortex was identified on the substrate.
\end{abstract}

Keywords: Numerical simulation; Diamond growth; Hot-filament chemical vapour deposition

\section{INTRODUCTION}

In the past few years there has been considerable increase in the study of polycrystalline diamond film. The impetus for theses studies has been provided by the discovery that diamond film can be grown on a variety of substrates under metastable conditions [1-3]. Diamond film have now been grown using a variety of deposition techniques and under a wide range of conditions. Diamond is a material of many unique properties. It is de hardest substance known to man, has the highest thermal conductivity of any known material at room temperature, is transmissive over a wide spectral range, highly inert and a good electrical insulator. Its properties have created potential opportunities for diamond in tribological, optical, microelectronics, thermal management and coating applications among other [2, 3]. Many of these potential applications can be realized by using diamond films obtained from chemical vapour deposition. Since, in most of these methods diamond films are deposited in sub atmospheric pressures and relatively low substrate temperatures, it is anticipated that many of the unique mechanical and optoelectronic properties of diamond can now be commercially exploded [4]. This work is based in the technique of chemical vapour deposition (CVD). In the CVD reactor a mixture of the gases is introduced, cross de activation source (filament) where the reaction take place (reaction zone) and produce the precursor species that are transported to the surface of the growth and then react to form diamond. The reactor geometry is shown in the Fig. 1.

The CVD is an important process for fabricating microelectronic circuits. A wide variety of semiconductors can be made using this process, and a large degree of control can be exerted over the deposition by altering the composition and concentration of active species in the gas phase. Individual layers of material can be deposited on a substrate by introducing various active species into the gas stream in the desired sequence and the rates of deposition with CVD can be of the order of microns/minute. Although, the potential exists for obtaining uniform deposition, the deposition process is dependent on the flow and the heat transfer from filament and substrate to the gas. The complexity of CVD processes is caused by the interdependence of the three main stages which determine the deposition: transport of the reactive species to the surface, chemical reaction on or near the surface and the transport of the reaction products from the surface [5]. The flow of the gas plays an important role controlling the rate and uniformity of deposition, because it governs the supply of reactants to the substrate that may be limited by mass transport. In this paper we analyze the fluid flow and the temperature distribution on the substrate. The computational task involves solution of a set of partial differential equations representing the essential physical features of the process.

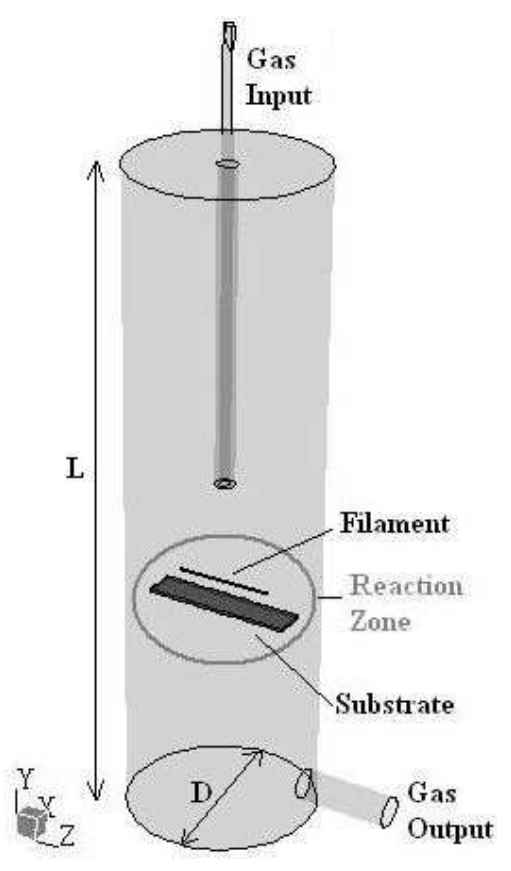

FIG. 1: Reactor geometry. 


\section{MATHEMATICAL MODEL AND CALCULATION METHOD}

The mathematical model can be described by the governing partial differential equations, i.e., the equations of conservation of momentum, mass, and heat. For a concrete reactor a 3D model was solved together with other physical input necessary to define the system adequately.

Continuity equation:

$$
\nabla \cdot(\rho \vec{v})=0
$$

Momentum equation:

$\nabla \cdot(\rho \vec{v} \vec{v})=\nabla \cdot \mu\left[(\nabla \vec{v})+(\nabla \vec{v})^{T}\right]+\nabla\left[\left(\mu^{\prime}-\frac{2}{3} \mu \nabla \cdot \vec{v}\right)\right]-\nabla p+\rho \vec{g}$

Energy equation:

$$
C_{p} \nabla \cdot(\rho T \vec{v})=\lambda\left(\nabla^{2} T\right)
$$

Where: $\rho$ is the density of the fluid, $v$ the velocity vector, $\mathrm{Cp}$ the specific heat, $\mathrm{T}$ the temperature, $\lambda$ the diffusion coefficient and $\mu$ the viscosity coefficient.

The following assumptions are made for the model formulation: (1) the flow of the gas mixture in the reactor is laminar. This is a realistic assumption, since flow in CVD reactor is normally slow and laminar to insure uniform film growth. (2) Binary diffusion coefficients are used in the hydrogen carrier gas mixture. This assumption is valid, because the more conventional techniques frequently utilizes $\mathrm{CH}_{4}$ as a carbonbearing feedstock and dilute this gas with large amounts of $\mathrm{H}_{2}$ to achieve inlet gas mixtures containing less than $1 \mathrm{~mol}$ $\% \mathrm{CH}_{4}$.(3) No chemical reaction takes place in this model. The calculations were performed with the geometry shown in Fig. 1. In the calculation a single straight filament of the diameter 0.25 microns was held $7 \mathrm{~mm}$ above the substrate. The temperature of the substrate and filament were maintained at $1000 \mathrm{~K}$ and $2500 \mathrm{~K}$ respectively. The operating pressure was $4.0 \cdot 10^{3} \mathrm{~Pa}$ (30 Torr) and the walls were maintained at the temperature $328 \mathrm{~K}$. The gas flow rate is $\approx 100 \mathrm{~cm}^{3} \mathrm{~min}^{-1}$. The simulation was performed using CFX [6], a commercial code which employs finite volume. The mesh grid used has $2 \cdot 10^{6}$ nodes with tetrahedral elements. Fig. 2 shows the superficial mesh used in the discretization of the equations. Fig. 3 shows $\mathrm{x}-\mathrm{y}$ plane. Fig. 4 shows in details the grid mesh used between the filament and substrate.

\section{RESULTS AND DISCUSSION}

When a mixture of hydrogen and methane comes near the hot filament, the temperature and consequently its composition changes. Temperature, pressure and flow composition are very important factors responsible for the amount of various

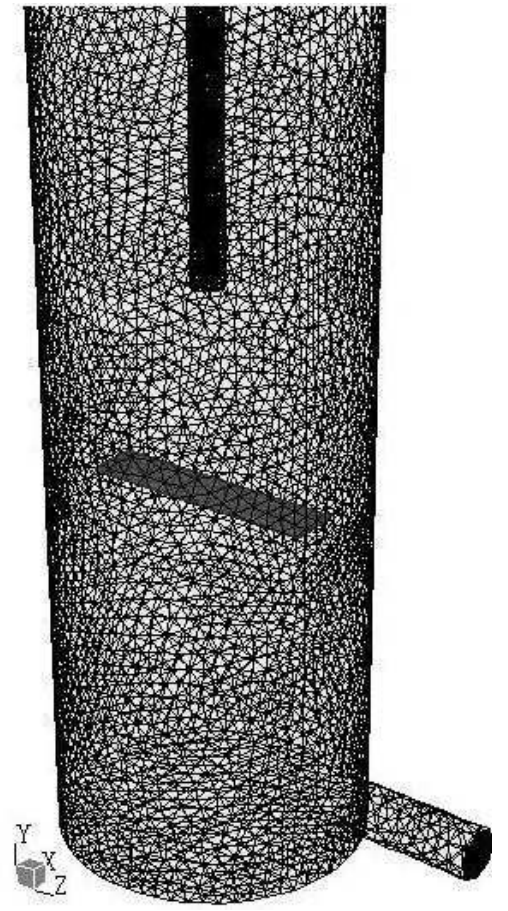

FIG. 2: Superficial mesh grid.

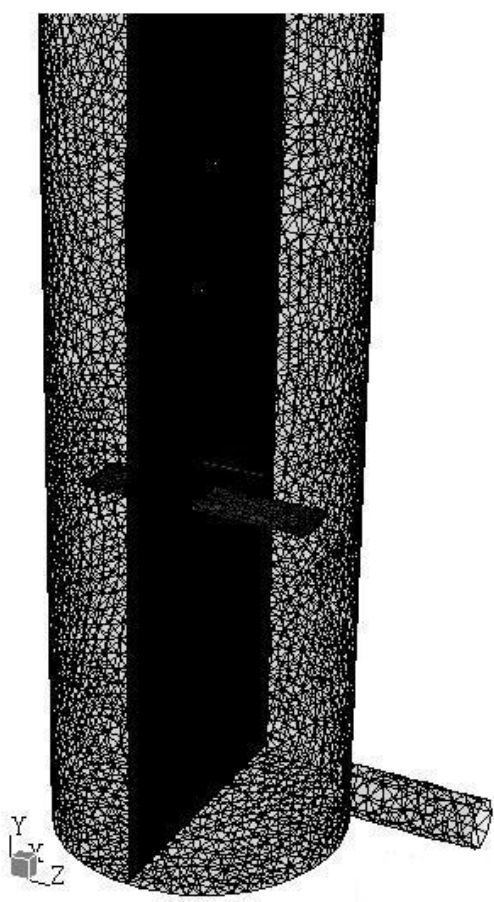

FIG. 3: x-y plane of the reactor geometry.

species formed near the filament. Fig. 5 shows the temperature distribution near the filament and substrate. The streamlines are slightly compressed near the filament while streamlines are relatively spread out in the direction of the substrate. This may suggest that the gas flow has an insignificant influence on the temperature distribution and that conduction is the 


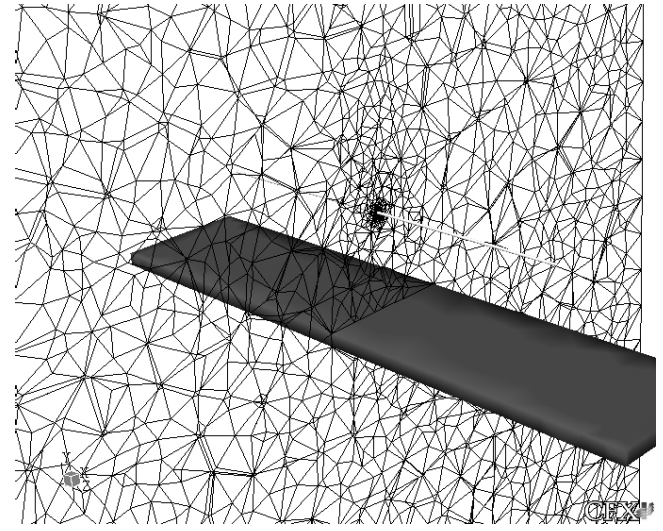

FIG. 4: Reaction zone of the grid mesh (detail of the substrate filament).

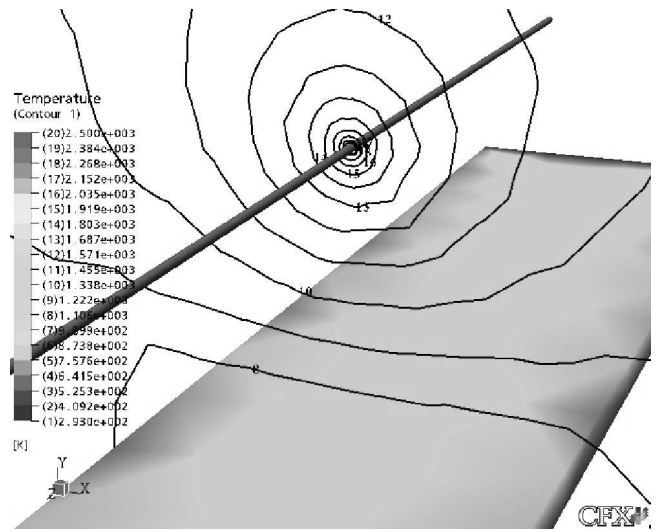

FIG. 5: Temperature distribution in the reaction zone.

primary factor in establishing the temperature field [7]. Fig. 6 shows a comparison of a calculated temperature and two experimental results. It is observed that the computed values are in good agreement with the experimental observations $[4,8]$. In the Fig. 7 is showed the velocity field profile. A pair of the vortex appears beside the filament and indicates the pres-

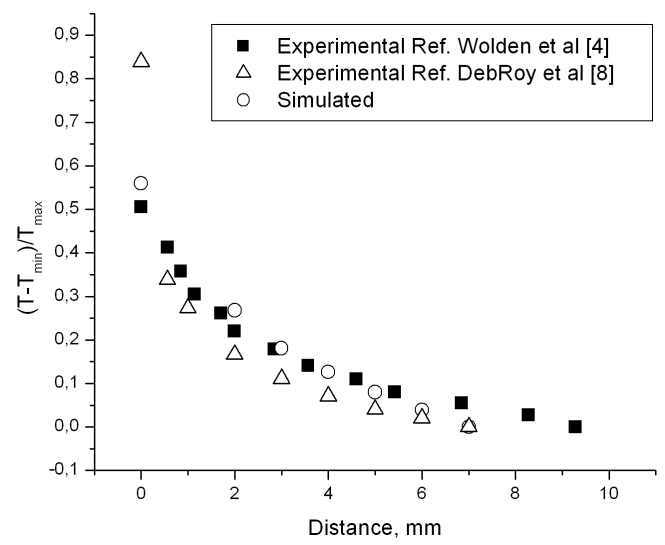

FIG. 6: Temperature profile between filament and substrate, comparison of simulated temperature with experimental results.

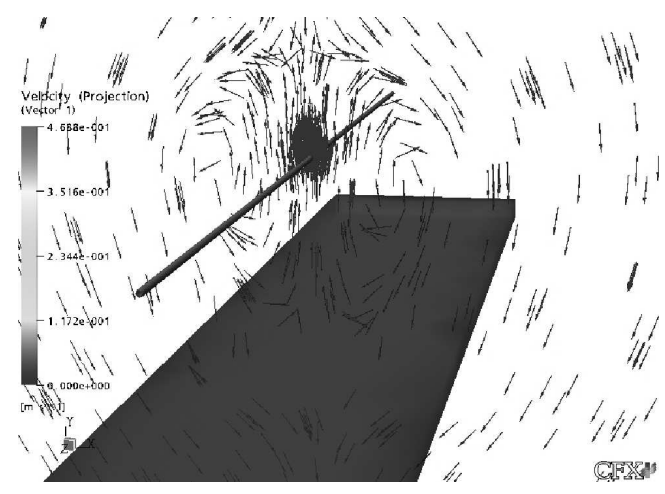

FIG. 7: The velocity field profile in the reaction zone.

ence of natural convection. With the help of a dimensionless number, the Peclet number for mass transfer, it is possible to understand the importance of the mass transport by convection and diffusion. The Peclet number is defined as the ratio of convective mass transfer to diffusive mass transfer and is given by $\mathrm{Pe}=\mathrm{u} \alpha / \mathrm{D}_{i j}$. Where $\mathrm{u}$ is the average velocity, $\alpha$ is a characteristic length, and $\mathrm{D}_{i j}$ is the diffusion coefficient and depends on the nature of the species, temperature, pressure and composition. The calculation of $\mathrm{D}_{i j}$ takes into account the assumption (2) made above. From the Fig. 7 is also possible to estimate the velocity near the filament $\approx 0.02 \mathrm{~m} / \mathrm{s}$. Using this velocity as average velocity, the characteristic length as the distance between the filament and substrate, $7 \mathrm{~mm}$, and the average temperature between the substrate and filament, $1750 \mathrm{~K}$, the Peclet number calculated for the system was $\approx$ $4 \cdot 10^{-4}$. This value indicates that diffusive mass transport is the dominant mechanism. A close look in Fig. 7 shows that the vortex velocity is too small to contribute with the mass transport. The Ref. [8-9] investigated the role of fluid flow and heat transfer in determining the quality of the diamond film. In these papers, experiments were conducted with various flow configurations. The authors have concluded that the convection play no role in the rates deposition. But this papers does not show the vortex caused by the natural convection and also do not measure the velocity of the vortex to verify the effective contribution.

\section{CONCLUSION}

The vortex due to natural convection is verified in this simulation. The results agree with the Peclet number and indicated that convective mass transfer is an unimportant effect to the growth of diamond. In Ref. 4 an especial designed gas flow configuration was planned to verify the importance of the convective flow in the CVD reactor. The results show no changes in the rates of deposition of diamond film, which agree with the simulations, showed in this paper. From the simulation is possible to obtain the profile temperature and compare with experimental results with a good agreement. This paper also shows that the CFX software is a powerful tool to simulate complex geometries. 


\section{Acknowledgement}

We are gratefully to FAPESP and CNPq, by the financial support of this work, under contract $n^{o} 02 / 12578-2$ and $n^{o}$
140243/2004-6 respectively.
[1] J. C. Angus and C. C. Hayman, Science. 241, 913 (1988).

[2] P. W. May, Phil. Trans. R. Soc. Lond. A. 358, 473 (2000).

[3] S.-T. Lee, Z. Lin and X. Jiang, Mater. Sci. Eng. 25, 123 (1999).

[4] C. Wolden and K. K. Gleason, Appl. Phys. Lett. 62, 2329 (1993).

[5] G. Wahl, Thin Solid Films. 40, 13 (1977).

[6] ANSYS Company, CFX-5 Solver Theory Manual CFX Ltd.,
ANSYS Company, Oxfordshire (2003).

[7] K. Tankala and T. DebRoy, J. Appl. Phys. 72, 712 (1992).

[8] K. Tankala and T. DebRoy, Surf. Coat. Technol. 62, 349 (1993).

[9] T. DebRoy, K. Tankala, W. A. Yarbrouugh and R. Messier, J. Appl. Phys. 68, 2424 (1990). 